\title{
Hall polynomials and the Gabriel-Roiter submodules of simple homogeneous modules
}

\author{
Csaba Szántó and István Szöllősi
}

\begin{abstract}
Let $k$ be an arbitrary field and $Q$ be an acyclic quiver of tame type (that is, of type $\left.\tilde{A}_{n}, \tilde{D}_{n}, \tilde{E}_{6}, \tilde{E}_{7}, \tilde{E}_{8}\right)$. Consider the path algebra $k Q$, the category of finite-dimensional right modules mod- $k Q$, and the minimal positive imaginary root of $Q$, denoted by $\delta$. In the first part of the paper, we deduce that the Gabriel-Roiter (GR) inclusions in preprojective indecomposables and homogeneous modules of dimension $\delta$, as well as their GR measures are field independent (a similar result due to Ringel being true in general over Dynkin quivers). Using this result, we can prove in a more general setting a theorem by Bo Chen which states that the GR submodule $P$ of a homogeneous module $R$ of dimension $\delta$ is preprojective of defect -1 and so the pair $(R / P, P)$ is a Kronecker pair. The generalization consists in considering the originally missing case $\tilde{E}_{8}$ and using arbitrary fields (instead of algebraically closed ones). Our proof is based on the idea of Ringel (used in the Dynkin quiver context) of comparing all possible Hall polynomials with the special form they take in case of a GR inclusion. For this purpose, we determine (with the help of a program written in GAP) a list of tame Hall polynomials which may have further interesting applications.
\end{abstract}

\section{Introduction}

Classical Hall algebras associated with discrete valuation rings were introduced by Steinitz and Hall to provide an algebraic approach to the classical combinatorics of partitions. The multiplication is given by classical Hall polynomials which play an important role in the representation theory of the symmetric groups and the general linear groups (see [9], the famous book by MacDonald devoted to its properties). In 1990, Ringel defined Hall algebras for a large class of rings, namely finitary rings, including in particular path algebras of quivers over finite fields. In general, these Ringel-Hall algebras are not commutative, in contrast with the classical ones (which correspond to the one-loop quiver in this context). In case of Ringel-Hall algebras associated to quivers, we know due to Ringel and Green that the subalgebra generated by the simple modules is up to a minor modification the positive part of the corresponding Drinfeld-Jimbo quantum group.

For Ringel-Hall algebras corresponding to Dynkin quivers (that is, of type $A_{n}, D_{n}, E_{6}, E_{7}, E_{8}$ ) and tame quivers (that is, of type $\tilde{A}_{n}, \tilde{D}_{n}, \tilde{E}_{6}, \tilde{E}_{7}, \tilde{E}_{8}$ ), it was proved by Ringel and Hubery that the structure constants of the multiplication are again polynomials in the number of elements of the base field. These are the generalized Hall polynomials, which will be referred simply as Hall polynomials throughout the paper. If we look at Hall polynomials associated to indecomposable modules, then the classical ones are just 0 or 1 and those appearing in the Dynkin case are also known and have degree up to 5 . However, we do not have too much information about them in the tame case. One of the results of the paper is a list of some new Hall polynomials over tame quivers.

Received 28 September 2013; revised 19 June 2014; published online 29 December 2014

2010 Mathematics Subject Classification 16G20 (primary).

The first author was supported by the Bolyai Scholarship of the Hungarian Academy of Sciences and Romanian Grant ID-PCE-2012-4-0100, the second author by the Romanian Grant RU-TE-2009-1-ID 303. 
Hall polynomials appear in various contexts: as mentioned above, they are the structure constants of quantum groups, they are used in the theory of cluster algebras and they can also be used successfully to investigate the structure of the module category. In this direction, the main results of the paper describe properties of Gabriel-Roiter (GR) submodules of some specific modules, using Hall polynomials in the proofs.

The GR measure was introduced by Gabriel in order to give a combinatorial interpretation of the induction scheme used by Roiter in his proof of the first Brauer-Thrall conjecture. Ringel used it as a foundation tool for the representation theory of artin algebras. Consider a finite-dimensional algebra and its finite length modules. If $M$ is an indecomposable module that is not simple, then it has GR submodules. These are indecomposable submodules $U$ satisfying a certain maximality condition, with length $|U|$ determined by $M$ and with $M / U$ indecomposable. The GR measure $\mu(M)$ of $M$ is the set of lengths $\left\{\left|M_{1}\right|, \ldots,\left|M_{t}\right|\right\}$ where $M_{1}$ is simple, $M_{t}=M$ and $M_{i-1}$ is a GR submodule of $M_{i}$. A GR inclusion is a monomorphism whose image is a GR submodule in the codomain.

Let $Q$ be a tame quiver (without oriented cycles) with vertex set $Q_{0}$, let $k$ be a field and mod- $k Q$ be the category of finite-dimensional right modules over the path algebra $k Q$. It is well known that the dimension vectors of indecomposable modules are just the positive roots of the corresponding Kac-Moody root system (which depends only on $Q$ and is a subset of $\mathbb{Z} Q_{0}$, the free abelian group with basis $Q_{0}$ ). Because $Q$ is tame, there exists a minimal positive imaginary root $\delta$ which defines a linear function $\partial$ on $\mathbb{Z} Q_{0}$, called the defect. More precisely, $\partial x=\langle\delta, x\rangle$, using the Euler form of $Q$. Looking at the defects of the dimensions, the indecomposables are either preprojectives (having negative defect), or preinjectives (having positive defect) or regulars (having zero defect). Note that a preprojective (preinjective) is uniquely determined (up to isomorphism) by its dimension vector, which is a positive real root $x$. Hence we may use the notation $P(x, k)$. The family of regular indecomposable modules with dimension $\delta$ that are homogeneous (that is, isomorphic with their Auslander-Reiten translate) play an important role in the global structure of mod- $k Q$. We will call them simply homogeneous modules with dimension $\delta$.

In our first two theorems (Theorems 3.8 and 3.10), we prove that in the case when $Q$ is tame without oriented cycles, the GR inclusions in preprojective indecomposables and homogeneous modules of dimension $\delta$ as well as their GR measures are field independent. A similar result for Dynkin quivers was obtained by Ringel in [13]. More precisely, our first theorem asserts that the GR measure of $P(y, k)$ is independent from $k$. Moreover, if $P(x, k) \rightarrow P(y, k)$ is a GR inclusion, then the roots $x$ depend also only on the root $y$ and not on $k$. The second theorem claims that GR measures of non-isomorphic homogeneous modules of dimension $\delta$ are equal and field independent. For a GR inclusion $P(x, k) \rightarrow R$, where $R$ is homogeneous of dimension $\delta$, the root $x$ is independent from $k$ and also from the isomorphism class of $R$. In order to prove these theorems, we use Ringel's ideas from [13], Chen's results from [5], Hall polynomials and results from algebraic geometry.

As an application of the theorems above, we can prove a result by Chen in $[6]$ in a more general context: our result is valid also for the case $\tilde{E}_{8}$ (this case is missing from [6]) and it is field independent (in [6] $k$ is algebraically closed). More precisely, we prove in Theorem 5.1 that a GR submodule $P$ of a homogeneous module $R$ of dimension $\delta$ has defect -1 . As a consequence, we obtain a Kronecker pair $(R / P, P)$ and in this way we can embed the module category of the Kronecker algebra into mod- $k Q$, by sending the simple projective to $P$ and the simple injective to $R / P$.

The proof of the theorem above follows the idea of Ringel from [13]: one compares all possible Hall polynomials with the special form they take in case of a GR inclusion. We have developed a GAP program in order to obtain the list of tame Hall polynomials corresponding to exact sequences of the form $0 \rightarrow P \rightarrow R \rightarrow I \rightarrow 0$, where $P$ is a preprojective, $I$ is a preinjective indecomposable and $R$ is a homogeneous module of dimension $\delta$ (see Theorem 4.2). It is 
surprising that there are only six different polynomials depending only on the defect of $P$ (and not even on the type of the tame quiver).

\section{Preliminaries}

First of all, we list some needed facts related to representations of tame quivers. For a detailed description, we refer the reader to $[\mathbf{1}, \mathbf{2}, \mathbf{7}]$.

Let $Q=\left(Q_{0}, Q_{1}\right)$ be a tame quiver without oriented cycles. Suppose that the vertex set $Q_{0}$ has $n$ elements and for an arrow $\alpha \in Q_{1}$ we denote by $t(\alpha), h(\alpha) \in Q_{0}$ the tail and head of $\alpha$. The Euler form of $Q$ is a bilinear form on $\mathbb{Z} Q_{0} \cong \mathbb{Z}^{n}$ given by $\langle x, y\rangle=\sum_{i \in Q_{0}} x_{i} y_{i}-$ $\sum_{\alpha \in Q_{1}} x_{t(\alpha)} y_{h(\alpha)}$. Its quadratic form $q_{Q}$ (called Tits form) is independent from the orientation of $Q$ and in the tame case it is positive semidefinite with radical $\mathbb{Z} \delta$, where $\delta$ is a minimal positive imaginary root of the corresponding Kac-Moody root system. The defect of $x \in \mathbb{Z} Q_{0}$ is then $\partial x=\langle\delta, x\rangle$.

Let $k$ be a field. The category $\bmod -k Q$ will be identified with the category rep- $k Q$ of the finite-dimensional $k$-representations of the quiver. We will denote by $[M]$ the isomorphism class of the module $M$, by $\underline{\operatorname{dim}} M \in \mathbb{Z} Q_{0} \cong \mathbb{Z}^{n}$ its dimension vector and by $\partial M=\partial(\underline{\operatorname{dim}} M)$ the defect of $M$. As mentioned in the introduction, the indecomposable modules in mod- $k Q$ are of three types: preprojectives (having negative defect), preinjectives (having positive defect) and regulars (having zero defect).

For $P$ preprojective (that is, with all its indecomposable components preprojective), $I$ preinjective and $R$ regular module, we have $\operatorname{Hom}(R, P)=\operatorname{Hom}(I, P)=\operatorname{Hom}(I, R)=$ $\operatorname{Ext}^{1}(P, R)=\operatorname{Ext}^{1}(P, I)=\operatorname{Ext}^{1}(R, I)=0$. It follows that the submodules of a preprojective module are always preprojective and a submodule of a regular module cannot have preinjective components. Preprojective and preinjective indecomposables are exceptional (that is, their endomorphism space is one-dimensional and they have no self-extensions) and are determined up to isomorphism by their dimension vector, which is a positive real root of the root system of $Q$. Note also that the possible defects of a preprojective indecomposable are -1 in the $\tilde{A}_{n}$ case, -1 or -2 in the $\tilde{D}_{n}$ case, $-1,-2$ or -3 in the $\tilde{E}_{6}$ case, ranging from -4 to -1 in the $\tilde{E}_{7}$ case and ranging from -6 to -1 in the $\tilde{E}_{8}$ case.

The category of regular modules is an abelian, exact subcategory which decomposes into a direct sum of serial categories with Auslander-Reiten quiver of the form $Z A_{\infty} / m$, called tubes of rank $m$. These tubes are indexed by the points of the projective line $\mathbb{P}_{k}^{1}$, the degree of a point $a \in \mathbb{P}_{k}^{1}$ being denoted by $\operatorname{deg} a$. A tube of rank 1 is called homogeneous, otherwise it is called non-homogeneous. We have at most three non-homogeneous tubes indexed by points $a$ of degree $\operatorname{deg} a=1$. All the other tubes are homogeneous. We assume that the non-homogeneous tubes are labelled by some subset of $\{0,1, \infty\}$, whereas the homogeneous tubes are labelled by the closed points of the scheme $\mathbb{H}_{k}=\mathbb{H}_{\mathbb{Z}} \otimes k$ for some open integral subscheme $\mathbb{H}_{\mathbb{Z}} \subset \mathbb{P}_{\mathbb{Z}}^{1}$. Let $\mathbb{X}_{k} \subseteq \mathbb{H}_{k}$ be the set of points of degree 1 . The indecomposables on a homogeneous tube labelled by $a \in \mathbb{H}_{k}$ are denoted by $R^{k}(1, a) \subset R^{k}(2, a) \subset \ldots$. For a partition $\lambda=\left(\lambda_{1}, \ldots, \lambda_{n}\right)$, let $R^{k}(\lambda, a)=R^{k}\left(\lambda_{1}, a\right) \oplus \cdots \oplus R^{k}\left(\lambda_{n}, a\right)$. Note that the homogeneous modules of dimension $\delta$ mentioned in the introduction are up to isomorphism the regular simples $R^{k}(1, a)$, with $a \in \mathbb{X}_{k}$. For simplicity, we will denote them by $R^{k}(a)$. Note that $\operatorname{dim}_{k} \operatorname{End}\left(R^{k}(a)\right)=1$.

We will describe now the so-called decomposition symbol and class used by Hubery in [8]. A module without homogeneous regular components can be described combinatorially in a field independent manner, using a system of positive real roots together with the dimensions of quasisocles for the non-homogeneous regular components of dimension $t \delta$. We denote this system by $\mu$ and let $M(\mu, k)$ be the corresponding unique module in mod- $k Q$ (up to isomorphism). A Segre symbol is a multiset $\sigma=\left\{\left(\lambda^{1}, d_{1}\right), \ldots,\left(\lambda^{r}, d_{r}\right)\right\}$, where $\lambda^{i}$ are partitions and $d_{i} \in \mathbb{N}^{*}$. It will describe the homogeneous regular components of the module. Using the definitions 
above, a decomposition symbol is pair $\alpha=(\mu, \sigma)$. Given a decomposition symbol $\alpha=(\mu, \sigma)$ and a field $k$, we define the decomposition class $S(\alpha, k)$ to be the set of isomorphism classes of modules of the form $M(\mu, k) \oplus R$, where $R=R^{k}\left(\lambda^{1}, a_{1}\right) \oplus \cdots \oplus R^{k}\left(\lambda^{r}, a_{r}\right)$ for some distinct points $a_{1}, \ldots a_{r} \in \mathbb{H}_{k}$ such that $\operatorname{deg} a_{i}=d_{i}$.

Note that for $k$ finite with $q$ elements $\left|\mathbb{X}_{k}\right|$ is either $q+1$ or $q$ or $q-1$ in the $\tilde{A}_{n}$ case and $q-2$ for other tame quivers. So if $k$ has two elements and the quiver is not of type $\tilde{A}_{n}$, then there are no homogeneous modules of dimension $\delta$. We also mention that for a decomposition symbol $\alpha$ the polynomial $n_{\alpha}(q)=|S(\alpha, k)|$ is strictly increasing in $q>1$.

For simplicity, denote by $x$ the decomposition symbol corresponding to a preprojective (preinjective) indecomposable given by the root $x$. Also, denote by $\delta$ the symbol corresponding to homogeneous modules of dimension $\delta$.

A pair of indecomposable modules $(Y, X)$ is called a Kronecker pair if they are exceptional, $\operatorname{Hom}(X, Y)=\operatorname{Hom}(Y, X)=\operatorname{Ext}^{1}(X, Y)=0$ and $\operatorname{dim}_{k} \operatorname{Ext}^{1}(Y, X)=2$. It is known due to Hubery (see $[\mathbf{8}]$ ) that in the tame case the Kronecker pairs are the pairs $(I, P)$, where $P$ is an indecomposable preprojective of defect -1 and $I$ is the indecomposable preinjective of dimension $\delta-\underline{\operatorname{dim} P}$ (and thus of defect 1 ).

Next we mention some needed facts about Ringel-Hall algebras. Suppose that $k$ is finite. We consider the rational Ringel-Hall algebra $\mathcal{H}(k Q)$ of the algebra $k Q$. Its $\mathbb{Q}$-basis is formed by the isomorphism classes $[M]$ from mod- $k Q$ and the multiplication is defined by $\left[N_{1}\right]\left[N_{2}\right]=$ $\sum_{[M]} F_{N_{1} N_{2}}^{M}[M]$. The structure constants $F_{N_{1} N_{2}}^{M}=\left|\left\{U \subseteq M \mid U \cong N_{2}, M / U \cong N_{1}\right\}\right|$ are called Ringel-Hall numbers.

Hubery proved the existence of Hall polynomials in tame cases with respect to the decomposition classes.

Theorem 2.1 [8]. Given decomposition symbols $\alpha, \beta$ and $\gamma$, there exists a rational polynomial $F_{\alpha \beta}^{\gamma}$ such that for any finite field $k$ with $|k|=q$,

$$
F_{\alpha \beta}^{\gamma}(q)=\sum_{\substack{A \in S(\alpha, k) \\ B \in S(\beta, k)}} F_{A B}^{C} \quad \text { for all } C \in S(\gamma, k) .
$$

We end this part with some facts on GR measures and submodules (see $[\mathbf{1 2}, \mathbf{1 3}]$ for details).

Let $\mathcal{P}\left(\mathbb{N}^{*}\right)$ be the set of all subsets of $\mathbb{N}^{*}$. A total order on $\mathcal{P}\left(\mathbb{N}^{*}\right)$ can be defined as follows: if $I, J$ are two different subsets of $\mathbb{N}$, then write $I<J$ if the smallest element in $(I \backslash J) \cup(J \backslash I)$ belongs to $J$. Using the total order above, for each $M \in \bmod -k Q$ let $\mu(M)$ be the maximum of the sets $\left\{\left|M_{1}\right|,\left|M_{2}\right|, \ldots,\left|M_{t}\right|\right\}$, where $M_{1} \subset M_{2} \subset \cdots \subset M_{t}$ is a chain of indecomposable submodules of $M$. Then $\mu(M)$ the GR measure of $M$. If $M \in \bmod -k Q$ is indecomposable and not simple, then an indecomposable submodule $U \subset M$ is called a GR submodule provided $\mu(M)=\mu(U) \cup\{|M|\}$, thus if and only if every proper submodule of $M$ has GR measure at most $\mu(U)$. A monomorphism $N \rightarrow M$ between two indecomposable modules is called GR inclusion if $\mu(M)=\mu(N) \cup\{|M|\}$ (that is, $N$ is isomorphic with a GR submodule of $M$ ). It is known that the factor of a GR inclusion is indecomposable. If $N \subset M$ is a GR inclusion, then the exact sequence $0 \rightarrow N \rightarrow M \rightarrow M / N \rightarrow 0$ will be called a GR exact sequence.

For two sets $I, J \in \mathcal{P}\left(\mathbb{N}^{*}\right)$, we say that $J$ starts with $I$ provided $I=J$ or $I \subset J$ and for all elements $a \in I$ and $b \in J \backslash I$ we have $a<b$. Finally, for $X, Y$ indecomposables let us denote by $\operatorname{Sing}(X, Y)$ the set of maps $X \rightarrow Y$ that are not monomorphisms.

We have the following lemma.

Lemma 2.2. Let $X, Y, Y_{1}, \ldots, Y_{t}$ and $Z$ be indecomposable modules.

(a) If $X$ is a proper submodule of $Y$, then $\mu(X)<\mu(Y)$. 
(b) Suppose there is a monomorphism $f: X \rightarrow Y_{1} \oplus \cdots \oplus Y_{t}$. If $\max \left\{\mu\left(Y_{i}\right)\right\}$ starts with $\mu(X)$ then there is some $j$ such that $\pi_{j} f$ is injective, where $\pi_{j}: Y_{1} \oplus \cdots \oplus Y_{t} \rightarrow Y_{j}$ is the canonical projection.

(c) If $X \rightarrow Y$ is a $G R$ inclusion, then $\operatorname{Sing}(X, Y)$ is a $k$-subspace of $\operatorname{Hom}(X, Y)$.

(d) Suppose that $k$ is finite with $q$ elements and let $X \rightarrow Y$ be a GR inclusion. Then the number $u_{X}^{Y}$ of submodules of $Y$ that are isomorphic to $X$ is $q^{s-r}\left(q^{h-s}-1\right) /\left(q^{e-r}-1\right)$, where $e=\operatorname{dim}_{k} \operatorname{End}(X), r=\operatorname{dim}_{k} \operatorname{radEnd}(X), h=\operatorname{dim}_{k} \operatorname{Hom}(X, Y), s=\operatorname{dim}_{k} \operatorname{Sing}(X, Y)$ and thus $h>s \geqslant r, e>r$.

\section{GR measure of preprojectives and homogeneous modules of dimension $\delta$}

The first aim of this section is to prove that the GR inclusions in preprojectives are field independent. In particular, the GR measure of preprojectives is also field independent. A similar statement is true for all the indecomposables in the Dynkin case (see [13]). Our approach for the tame case uses Ringel's ideas from [13] but also introduces some new concepts. Note that contrary to the Dynkin case, we cannot use Schofield short exact sequences.

First, we consider some conventions. Consider a representation $M$ of dimension $x=\left(x_{i}\right)_{i \in Q_{0}}$ such that its linear application $k^{x_{t(\alpha)}} \rightarrow k^{x_{h(\alpha)}}$ corresponding to the arrow $\alpha$ is given by the matrix $A_{\alpha}$ (in the canonical base). An endomorphism of this representation (using the canonical bases) can be identified with a collection $\left(X_{i}\right)_{i \in Q_{0}}$ of square matrices $X_{i}$ of dimension $x_{i}$ that satisfy the relations $A_{\alpha} X_{t(\alpha)}=X_{h(\alpha)} A_{\alpha}$. These relations induce a homogeneous linear system of equations with the unknowns being the elements of $X_{i}$. Denote by $A_{M}$ the matrix of this system. Trivially, we have $\operatorname{dim}_{k} \operatorname{End}(M)=\operatorname{corank} A_{M}$.

Let $k$ be a field with prime field $k_{0}, x$ be a positive real root such that $\partial x<0$ and $P(x, k)$ be the preprojective indecomposable representation over $k$ with dimension $x$ (unique up to isomorphism). In case we use the representation $P\left(x, k_{0}\right)$ over the prime field $k_{0}$ of $k$, we agree that the representation $P(x, k)$ is constructed in the following way: for $\alpha \in Q_{1}$ consider the linear application $k^{x_{t(\alpha)}} \rightarrow k^{x_{h(\alpha)}}$ having the same matrix in the canonical base as the linear application $k_{0}^{x_{t(\alpha)}} \rightarrow k_{0}^{x_{h(\alpha)}}$ in the representation $P\left(x, k_{0}\right)$. Note that $P(x, k)$ is an indecomposable representation over $k$ of dimension $x$ (since $A_{P(x, k)}=A_{P\left(x, k_{0}\right)}$, so $\left.\operatorname{dim}_{k} \operatorname{End}(P(x, k))=\operatorname{corank} A_{P(x, k)}=\operatorname{corank} A_{P\left(x, k_{0}\right)}=1=\operatorname{dim}_{k} \operatorname{End}\left(P\left(x, k_{0}\right)\right)\right)$. A second convention is that if we use the rational representation $P(x, \mathbb{Q})$ with rational matrices corresponding to the arrows (in the canonical base) $A_{\alpha}$, then for a big enough prime $p$ the representation $P\left(x, \mathbb{F}_{p}\right)$ has matrices $A_{\alpha} \bmod p$. Note also that this representation $P\left(x, \mathbb{F}_{p}\right)$ is indecomposable for $p$ big enough.

The conventions above and the fact that a rational matrix taken modulo a big enough prime keeps its rank imply the following lemma.

Lemma 3.1. (a) If there is a monomorphism $P\left(x, k_{0}\right) \rightarrow P\left(y, k_{0}\right)$, then there is a monomorphism $P(x, k) \rightarrow P(y, k)$.

(b) If there is a monomorphism $P(x, \mathbb{Q}) \rightarrow P(y, \mathbb{Q})$, then there is a monomorphism $P\left(x, \mathbb{F}_{p}\right) \rightarrow P\left(y, \mathbb{F}_{p}\right)$ for a prime $p$ big enough.

The next lemma is a straightforward generalization of the corresponding result by Ringel in the Dynkin case (see [13]).

LEMma 3.2. If there is a GR inclusion $P(x, k) \rightarrow P(y, k)$, then there is a monomorphism $P\left(x, k_{0}\right) \rightarrow P\left(y, k_{0}\right)$.

Using the existence of Hall polynomials in the tame case, we obtain the following lemma. 
Lemma 3.3. If there is a monomorphism $P(x, k) \rightarrow P(y, k)$ for $k$ finite, then there is a monomorphism $P\left(x, k^{\prime}\right) \rightarrow P\left(y, k^{\prime}\right)$ for $k^{\prime}$ finite and $\left|k^{\prime}\right|$ big enough.

Proof. Denote by $\alpha$ the decomposition symbol of the cokernel in the monomorphism $P(x, k) \rightarrow P(y, k)$. Using Theorem 2.1, there is a rational polynomial $F_{\alpha x}^{y}$ such that for any field $k^{\prime}$ with $q^{\prime} \geqslant q$ elements $F_{\alpha x}^{y}\left(q^{\prime}\right)=\sum_{A \in S\left(\alpha, k^{\prime}\right)} F_{A P\left(x, k^{\prime}\right)}^{P\left(y, k^{\prime}\right)}$. Due to our condition, $F_{\alpha x}^{y}$ is a nonzero polynomial, so for $q^{\prime}$ big enough $F_{\alpha x}^{y}\left(q^{\prime}\right)$ is also nonzero. This implies our statement.

Using all the lemmas above, we obtain the following proposition.

Proposition 3.4. (a) Consider a field $k$ and its prime field $k_{0}$. Then $\mu\left(P\left(x, k_{0}\right)\right)=$ $\mu(P(x, k))$, moreover we have a GR inclusion $P\left(x, k_{0}\right) \rightarrow P\left(y, k_{0}\right)$ if and only if we have a GR inclusion $P(x, k) \rightarrow P(y, k)$.

(b) Consider two fields $k, k^{\prime}$ with prime characteristic and a third field $k^{\prime \prime}$ of characteristic 0. Then $\mu(P(x, k))=\mu\left(P\left(x, k^{\prime}\right)\right) \geqslant \mu\left(P\left(x, k^{\prime \prime}\right)\right)=\mu(P(x, \mathbb{Q}))$, moreover we have a GR inclusion $P(x, k) \rightarrow P(y, k)$ if and only if we have a GR inclusion $P\left(x, k^{\prime}\right) \rightarrow P\left(y, k^{\prime}\right)$.

Proof. (a) If $\mu(P(x, k))=\left\{n_{1}, \ldots, n_{t}\right\}$, then (using the fact that submodules of preprojectives are preprojective) there is a sequence of GR inclusions $P\left(x_{1}, k\right) \rightarrow \cdots \rightarrow P\left(x_{t}, k\right)=$ $P(x, k)$ with $\left|P\left(x_{i}, k\right)\right|=n_{i}$. By Lemma 3.2, there is a chain of monomorphisms $P\left(x_{1}, k_{0}\right) \rightarrow$ $\cdots \rightarrow P\left(x_{t}, k_{0}\right)=P\left(x, k_{0}\right)$ with $\left|P\left(x_{i}, k_{0}\right)\right|=n_{i}$. It follows that $\mu(P(x, k)) \leqslant \mu\left(P\left(x, k_{0}\right)\right)$. In the same manner, using Lemma 3.1(a) we obtain that $\mu\left(P\left(x, k_{0}\right)\right) \leqslant \mu(P(x, k))$, so $\mu(P(x, k))=$ $\mu\left(P\left(x, k_{0}\right)\right)$.

Suppose now that we have a GR inclusion $P(x, k) \rightarrow P(y, k)$, so $\mu(P(y, k))=\mu(P(x, k)) \cup$ $\{|y|\}$. Then by Lemma 3.2 , we have a monomorphism $P\left(x, k_{0}\right) \rightarrow P\left(y, k_{0}\right)$. Moreover, using the first part of our statement we have $\mu\left(P\left(y, k_{0}\right)\right)=\mu(P(y, k))=\mu(P(x, k)) \cup\{|y|\}=$ $\mu\left(P\left(x, k_{0}\right)\right) \cup\{|y|\}$, which means that we have a GR inclusion $P\left(x, k_{0}\right) \rightarrow P\left(y, k_{0}\right)$. Conversely, we proceed in the same way.

(b) Using (a), we have $\mu(P(x, \mathbb{Q}))=\mu\left(P\left(x, k^{\prime \prime}\right)\right)$. Suppose that char $k=p$ and char $k^{\prime}=q$. Then again by $(\mathrm{a})$, we have $\mu(P(x, k))=\left(P\left(x, \mathbb{F}_{p}\right)\right)$ and $\mu\left(P\left(x, k^{\prime}\right)\right)=\mu\left(P\left(x, \mathbb{F}_{q}\right)\right)$. Using Lemma 3.3, one gets that $\mu\left(P\left(x, \mathbb{F}_{p}\right)\right) \leqslant \mu\left(P\left(x, \mathbb{F}_{q^{l}}\right)\right)$ for $l$ big enough. But as before $\mu\left(P\left(x, \mathbb{F}_{q^{l}}\right)\right)=\mu\left(P\left(x, \mathbb{F}_{q}\right)\right)$, so $\mu\left(P\left(x, \mathbb{F}_{p}\right)\right) \leqslant \mu\left(P\left(x, \mathbb{F}_{q}\right)\right)$, which implies (changing $p$ with $q$ ) that $\mu\left(P\left(x, \mathbb{F}_{p}\right)\right)=\mu\left(P\left(x, \mathbb{F}_{q}\right)\right)$. By Lemma 3.1(b), we obtain that $\mu(P(x, \mathbb{Q})) \leqslant \mu\left(P\left(x, \mathbb{F}_{r}\right)\right)$ for a big enough prime $r$.

Suppose now that we have a GR inclusion $P(x, k) \rightarrow P(y, k)$, so $\mu(P(y, k))=\mu(P(x, k)) \cup$ $\{|y|\}$. Then by Lemma 3.2, we have a monomorphism $P\left(x, \mathbb{F}_{p}\right) \rightarrow P\left(y, \mathbb{F}_{p}\right)$, so by Lemma 3.3 we also have a monomorphism $P\left(x, \mathbb{F}_{q^{l}}\right) \rightarrow P\left(y, \mathbb{F}_{q^{l}}\right)$ for $l$ big enough. Moreover, using the first part of our statement we have $\mu\left(P\left(y, \mathbb{F}_{q^{l}}\right)\right)=\mu(P(y, k))=\mu(P(x, k)) \cup\{|y|\}=\mu\left(P\left(x, \mathbb{F}_{q^{l}}\right)\right) \cup$ $\{|y|\}$, which means that we have a GR inclusion $P\left(x, \mathbb{F}_{q^{l}}\right) \rightarrow P\left(y, \mathbb{F}_{q^{l}}\right)$. By Lemmas 3.1(a) and 3.2, this implies a monomorphism $P\left(x, k^{\prime}\right) \rightarrow P\left(y, k^{\prime}\right)$ which is in fact a GR inclusion since $\mu\left(P\left(y, k^{\prime}\right)\right)=\mu(P(y, k))=\mu(P(x, k)) \cup\{|y|\}=\mu\left(P\left(x, k^{\prime}\right)\right) \cup\{|y|\}$.

The proposition above and Lemma 2.2 together imply the following proposition.

Proposition 3.5. Let $k^{\prime}$ be a finite field with $q^{\prime}$ elements. If we have a GR inclusion $P\left(x, k^{\prime}\right) \rightarrow P\left(y, k^{\prime}\right)$, then there is a prime power $q_{0}$ such that for every finite field $k$ with $q \geqslant q_{0}$ elements it is true that

$$
u_{P(x, k)}^{P(y, k)}=\frac{q^{h}-q^{s}}{q-1}=q^{s}\left(q^{h-s-1}+\cdots+q+1\right),
$$

where $h=\operatorname{dim}_{k} \operatorname{Hom}(P(x, k), P(y, k))>s=\operatorname{dim}_{k} \operatorname{Sing}(P(x, k), P(y, k))$ are field independent. 
Proof. By Theorem 2.1, there is a rational polynomial $f=\sum_{\alpha} F_{\alpha x}^{y}$, where $\alpha$ runs over all decomposition symbols of dimension $y-x$. We also know that there is a prime power $q_{0}$ such that $n_{\alpha}(q)=|S(\alpha, k)| \neq 0$ for all decomposition symbols $\alpha$ of dimension $y-x$ and every finite field $k$ with $q \geqslant q_{0}$ elements.

Since we have a GR inclusion $P\left(x, k^{\prime}\right) \rightarrow P\left(y, k^{\prime}\right)$, then using the proposition above we have a GR inclusion $P(x, k) \rightarrow P(y, k)$ for every finite field $k$ and by Lemma 2.2(c) and (d)

$$
u_{P(x, k)}^{P(y, k)}=q^{s_{k}}\left(q^{h-s_{k}-1}+\cdots+q+1\right),
$$

where $h=\operatorname{dim}_{k} \operatorname{Hom}(P(x, k), P(y, k))=\langle x, y\rangle$ is field independent (preprojectives being directing) and $s_{k}=\operatorname{dim}_{k} \operatorname{Sing}(P(x, k), P(y, k))$. Obviously, we also have that $u_{P(x, k)}^{P(y, k)}=f(q)$ for a finite field $k$ with $q \geqslant q_{0}$ elements. Since $s_{k}<h$, there is a value $s<h$ such that

$$
u_{P(x, k)}^{P(y, k)}=q^{s}\left(q^{h-s-1}+\cdots+q+1\right)
$$

for infinitely many values $q$, but this means that $f=\left(X^{h}-X^{s}\right) /(X-1)$.

Our next proposition uses a classical lemma taken from [4] (see also [14]).

Lemma 3.6 [4]. Let $X$ be a variety defined over some ring of algebraic integers. We denote by $X(\mathbb{C})$ (respectively, $X\left(\mathbb{F}_{q}\right)$ ) the set of $\mathbb{C}$-points (respectively, $\mathbb{F}_{q}$-points) of $X$. Suppose that there exists a polynomial $f$ with integral coefficients such that $\left|X\left(\mathbb{F}_{q}\right)\right|=f(q)$ for infinitely many prime powers $q$. Then the Euler-Poincaré characteristic (with compact support) of $X(\mathbb{C})$ is given by $\chi(X(\mathbb{C}))=f(1)$.

Proposition 3.7. If there is a GR inclusion $P(x, k) \rightarrow P(y, k)$ for a finite field $k$, then there is a monomorphism $P(x, \mathbb{C}) \rightarrow P(y, \mathbb{C})$.

Proof. Due to Ringel (see $[\mathbf{1 1}]$ ), we know that $P(x, \mathbb{C})$ and $P(y, \mathbb{C})$ are tree modules, so we can suppose that their matrices corresponding to the arrows contain only 0 and 1 . These representations exist also over $\mathbb{Q}$ and modulo $p$ (with the same matrices) in case $p$ is a big enough prime. We fix such a $p$ from now on.

Define $X=\{N \in \bmod -\mathbb{Q} Q \mid N \leqslant P(y, \mathbb{Q}), N \cong P(x, \mathbb{Q})\}$. Since we have that $X=\{N \in$ $\left.\bmod -\mathbb{Q} Q \mid N \leqslant P(y, \mathbb{Q}), \underline{\operatorname{dim}} N=x, \operatorname{dim}_{k} \operatorname{End}(N)=1\right\}$, one can see that $X$ is a (locally closed) subvariety of the quiver Grassmannian $\operatorname{Gr}_{x}(P(y, \mathbb{Q}))=\{N \in \bmod -\mathbb{Q} Q \mid N \leqslant P(y, \mathbb{Q}), \underline{\operatorname{dim}} N=$ $x\}$. One can also see that $X$ has in fact a $\mathbb{Z}$-form (see [14]) and in this way $X\left(\mathbb{F}_{p^{l}}\right)=\{N \in$ $\left.\bmod -\mathbb{F}_{p^{l}} Q \mid N \leqslant P\left(y, \mathbb{F}_{p^{l}}\right), N \cong P\left(x, \mathbb{F}_{p^{l}}\right)\right\}$.

Since we have a GR inclusion $P(x, k) \rightarrow P(y, k)$ for a finite field $k$, then using Proposition 3.5 we obtain for $p$ big enough that

$$
\left|X\left(\mathbb{F}_{p^{l}}\right)\right|=u_{P\left(x, \mathbb{F}_{p^{l}}\right)}^{P\left(\mathbb{F}_{p^{\prime}}\right)}=p^{l s}\left(p^{l(h-s-1)}+\cdots+p^{l}+1\right),
$$

so using Lemma 3.6 we have that $\chi(X(\mathbb{C})) \neq 0$ which means that there is a monomorphism $P(x, \mathbb{C}) \rightarrow P(y, \mathbb{C})$.

Putting together all the pieces from above, we obtain our first main result.

Theorem 3.8. Consider two fields $k, k^{\prime}$. Then $\mu(P(x, k))=\mu\left(P\left(x, k^{\prime}\right)\right)$, moreover, we have a GR inclusion $P(x, k) \rightarrow P(y, k)$ if and only if we have a GR inclusion $P\left(x, k^{\prime}\right) \rightarrow P\left(y, k^{\prime}\right)$.

Proof. By Proposition 3.4, it is enough to consider the case when $k$ has characteristic 0 and $k^{\prime}$ characteristic $p$. We know already that $\mu(P(x, k)) \leqslant \mu\left(P\left(x, k^{\prime}\right)\right)$. Conversely, by Propositions 3.4 and 3.7 we have $\mu\left(P\left(x, k^{\prime}\right)\right)=\mu\left(P\left(x, \mathbb{F}_{p}\right)\right) \leqslant \mu(P(x, \mathbb{C}))=\mu(P(x, \mathbb{Q}))=$ 
$\mu(P(x, k))$. The second part of the statement follows in the same manner as in the proof of Proposition 3.4.

We consider now homogeneous modules of dimension $\delta$ denoted by $R^{k}(a)$, with $a \in \mathbb{X}_{k}$. We will prove that the GR measure of $R^{k}(a)$ and also the GR inclusions in $R^{k}(a)$ do not depend on $a$ and on the field $k$.

For the proof of the second main theorem, we need the following result from [5].

Lemma 3.9 [5]. If $P$ is a preprojective indecomposable and $R^{k}(a)$ is a homogeneous module of dimension $\delta$, then $\mu(P)<\mu\left(R^{k}(a)\right)$. Moreover, if $|P|<|\delta|$, then there is a monomorphism $P \rightarrow t R^{k}(a)$ for some $t \in \mathbb{N}^{*}$, so $P$ is cogenerated by $R^{k}(a)$.

Theorem 3.10. Consider the fields $k, k^{\prime}$ and the points $a \in \mathbb{X}_{k}, a^{\prime} \in \mathbb{X}_{k^{\prime}}$. Then we have $\mu\left(R^{k}(a)\right)=\mu\left(R^{k^{\prime}}\left(a^{\prime}\right)\right)$. Moreover, we have a GR inclusion $P(x, k) \rightarrow R^{k}(a)$ if and only if we have a GR inclusion $P\left(x, k^{\prime}\right) \rightarrow R^{k^{\prime}}\left(a^{\prime}\right)$.

Proof. Suppose that $\mu\left(R^{k}(a)\right)<\mu\left(R^{k^{\prime}}\left(a^{\prime}\right)\right)$ and denote by $P(x, k)$ and $P\left(x^{\prime}, k^{\prime}\right)$ the GR submodules of $R^{k}(a)$ and $R^{k^{\prime}}\left(a^{\prime}\right)$. On one hand, we have that $\mu(P(x, k)) \cup\{|\delta|\}<$ $\mu\left(P\left(x^{\prime}, k^{\prime}\right)\right) \cup\{|\delta|\}$ which implies $\mu(P(x, k))<\mu\left(P\left(x^{\prime}, k^{\prime}\right)\right)$. On the other hand, using Theorem 3.8 and the proposition above we obtain $\mu\left(P\left(x^{\prime}, k^{\prime}\right)\right)=\mu\left(P\left(x^{\prime}, k\right)\right)<\mu\left(R^{k}(a)\right)=$ $\mu(P(x, k)) \cup\{|\delta|\}$, a contradiction since all the lengths in $\mu(P(x, k))$ and $\mu\left(P\left(x^{\prime}, k^{\prime}\right)\right)$ are smaller then $|\delta|$.

Suppose now that we have a GR inclusion $P\left(x, k^{\prime}\right) \rightarrow R^{k^{\prime}}\left(a^{\prime}\right)$. This means using the results above that $\mu\left(R^{k}(a)\right)=\mu\left(R^{k^{\prime}}\left(a^{\prime}\right)\right)=\mu\left(P\left(x, k^{\prime}\right)\right) \cup\{|\delta|\}=\mu(P(x, k)) \cup\{|\delta|\}$. So one can see that $\mu\left(R^{k}(a)\right)$ starts with $\mu(P(x, k))$.

Since $|P(x, k)|=\left|P\left(x, k^{\prime}\right)\right|<|\delta|$, it follows by Lemma 3.9 that there is a monomorphism $P(x, k) \rightarrow t R^{k}(a)$ for some $t \in \mathbb{N}^{*}$. But then using Lemma 2.2(b), it follows that there is a monomorphism $P(x, k) \rightarrow R^{k}(a)$. However $\mu\left(R^{k}(a)\right)=\mu(P(x, k)) \cup\{|\delta|\}$, so this monomorphism is a GR inclusion.

The following corollary clarifies the form of the Hall polynomial corresponding to the GR inclusion $P(x, k) \rightarrow R^{k}(a)$.

Corollary 3.11. Let $k$ be a finite field with $q$ elements. Then $u_{P(x, k)}^{R^{k}(a)}=F_{\delta-x x}^{\delta}(q)=$ $\left(q^{-\partial x}-q^{s}\right) /(q-1)$ where $-\partial x=\langle x, \delta\rangle>s=\operatorname{dim}_{k} \operatorname{Sing}\left(P(x, k), R^{k}(a)\right)$ are field independent and independent of $a$.

Proof. Since we have a GR inclusion $P(x, k) \rightarrow R^{k}(a)$, then the factor is indecomposable, so it is isomorphic to $I(\delta-x, k)$. By Theorem 2.1 , there is a rational polynomial $F_{\delta-x x}^{\delta}$ such that $F_{\delta-x x}^{\delta}(q)=F_{I(\delta-x, k) P(x, k)}^{R^{k}(a)}$. Since by the previous theorem the GR inclusions in $R^{k}(a)$ do not depend on $a$ and on the field $k$, we obtain using Lemma 2.2(d) that for $q$ big enough $F_{\delta-x x}^{\delta}(q)=\left(q^{-\partial x}-q^{s_{k}}\right) /(q-1)$ (with $\left.s_{k}<-\partial x\right)$, which means that there is a value $s$ such that for infinitely many values $q$ we have $F_{\delta-x x}^{\delta}(q)=\left(q^{-\partial x}-q^{s}\right) /(q-1)$. But then $F_{\delta-x x}^{\delta}=$ $\left(X^{-\partial x}-X^{s}\right) /(X-1)$.

\section{Reflection functors and Hall polynomials}

In this section, we suppose that the tame quiver $Q$ is not of type $\tilde{A}_{n}$ (so it is a tree) and $k$ is a finite field with $q$ elements. 
Let $i$ be a sink in the quiver $Q$. Denote by $s_{i}$ the reflection induced by the vertex $i$, by $\sigma_{i} Q$ the quiver obtained by reversing all arrows involving $i$ and by $Q_{i}$ the quiver having the same underlying graph as $Q$ with all its edges pointing towards $i$ (so $i$ is the unique $\operatorname{sink}$ in $Q_{i}$ ). Let mod- $k Q\langle i\rangle$ be the full subcategory of modules not containing the simple module corresponding to the vertex $i$ as a direct summand.

We consider the reflection functors $S_{i}^{+}: \bmod -k Q \rightarrow \bmod -k \sigma_{i} Q$ and $S_{i}^{-}: \bmod -k \sigma_{i} Q \rightarrow$ $\bmod -k Q$. For all details concerning reflection functors, we refer the reader to [3]. It is well known that for an indecomposable $M$ we have $S_{i}^{+} M \neq 0$ if and only if $M \not S_{i}$. Moreover, in this case $S_{i}^{+} M$ is indecomposable and $\underline{\operatorname{dim}} S_{i}^{+} M=s_{i}(\underline{\operatorname{dim}} M)$. Also the functors $S_{i}^{+}, S_{i}^{-}$ induce quasi-inverse equivalences between mod- $k Q\langle i\rangle$ and mod- $k \sigma Q\langle i\rangle$. It is easy to see that for $M \in \bmod -k Q\langle i\rangle$ indecomposable, we have that $\partial S_{i}^{+} M=\partial M$ and if $R$ is a simple homogeneous (respectively, non-homogeneous) regular, then so is $S_{i}^{+} R$. Finally, note that for $M, N, L \in \bmod -k Q\langle i\rangle$ we have $F_{M N}^{L}=F_{S_{i}^{+} M S_{i}^{+} N^{*}}^{S^{+} L}$

Let $i$ be a sink in $Q$ and denote by $N_{i}$ the set of neighbours of $i$. Then there exists a sequence $i_{1}, \ldots, i_{t}$ of vertices of $Q$ different from $i$ and not in $N_{i}$ such that for each $s \in\{1, \ldots, t\}$ the vertex $i_{s}$ is a sink in $\sigma_{i_{s-1}} \cdots \sigma_{i_{1}} Q$ and $\sigma_{i_{t}} \cdots \sigma_{i_{1}} Q=Q_{i}$.

Let $R^{k}(a)$ and $R^{k}\left(a^{\prime}\right)$ be two homogeneous modules of dimension $\delta$ and $P(x, k)$ be an indecomposable preprojective and $I(\delta-x, k)$ be the corresponding indecomposable preinjective. Then by Theorem 2.1, we have a Hall polynomial $F_{\delta-x x}^{\delta}$ such that

$$
F_{I(\delta-x, k) P(x, k)}^{R^{k}(a)}=F_{I(\delta-x, k) P(x, k)}^{R^{k}\left(a^{\prime}\right)}=F_{\delta-x x}^{\delta}(q) .
$$

Let $e_{i}=(0, \ldots, 1, \ldots 0)$ (with 1 at the $i$ th place) be the dimension of the simple projective module corresponding to the unique sink $i$ in $Q_{i}$. We show that the Hall polynomials $F_{\delta-x x}^{\delta}$ over the quiver $Q$ are equal to special Hall polynomials $F_{\delta-e_{i} e_{i}}^{\delta}$ over unique sink quivers $Q_{i}$.

Proposition 4.1. There is a vertex $i\left(\right.$ with $\left.\delta_{i}=-\partial x\right)$ such that ${ }^{Q} F_{\delta-x x}^{\delta}={ }^{Q_{i}} F_{\delta-e_{i} e_{i}}^{\delta}$, where the first polynomial is taken over the quiver $Q$ and the second one over the quiver $Q_{i}$.

Proof. We know that there exists field independently a sequence $i_{1}, \ldots, i_{t}$ of vertices in $Q$ such that for each $s \in\{1, \ldots, t\}$ the vertex $i_{s}$ is a sink in $\sigma_{i_{s-1}} \cdots \sigma_{i_{1}} Q$ and $S_{i_{t}}^{+} \cdots S_{i_{1}}^{+} P(x, k)=$ $S^{\prime \prime}(i) \in \bmod -k Q^{\prime \prime}$ is a simple projective corresponding to the sink $i$ in $Q^{\prime \prime}=\sigma_{i_{t}} \cdots \sigma_{i_{1}} Q$. It follows that

$$
F_{I(\delta-x, k) P(x, k)}^{R^{k}(a)}=F_{S_{i_{s}}^{+} \cdots S_{i_{1}}^{+} I(\delta-x, k) S^{\prime \prime}(i)}^{S_{+}^{+} \cdots S^{+} R^{k}(a)}=F_{S_{i_{s}}^{+} \cdots S_{i_{1}}^{+} I(\delta-x, k) S^{\prime \prime}(i)}^{R^{\prime \prime}},
$$

where $R^{\prime \prime}$ is a homogeneous regular module in $\bmod -k Q^{\prime \prime}$ of dimension $\delta$. Also there is a sequence $j_{1}, \ldots, j_{r}$ of vertices in $Q^{\prime \prime}$ different from $i$ and not in $N_{i}$ such that for each $s \in\{1, \ldots, t\}$ the vertex $j_{s}$ is a sink in $\sigma_{j_{s-1}} \cdots \sigma_{j_{1}} Q^{\prime \prime}$ and $\sigma_{j_{r}} \cdots \sigma_{j_{1}} Q^{\prime \prime}=Q_{i}$. It follows that $S_{j_{r}}^{+} \cdots S_{j_{1}}^{+} S^{\prime \prime}(i)=S^{\prime}(i)$ is the simple projective in mod- $k Q_{i}$ corresponding to the unique sink $i$ in $Q_{i}$. The statement now follows using the same argument as above.

Using a computer program written in GAP (see [15]), we have computed the special Hall polynomials from above. The program computes the Ringel-Hall numbers over small finite fields and interpolates the Hall polynomials. Due to the particular orientation of $Q_{i}$, the low dimensions and the symmetries, only a few cases occur and thus the computing time is very short. It takes around $15 \mathrm{~min}$ to obtain the polynomial list in the following theorem. We should also remark that using our program we could reproduce Ringel's list of Hall polynomials in the Dynkin case (see [10]). Summarizing all above, we obtain the following theorem. 
Theorem 4.2. Let $x$ be a positive real root with $\partial x<0$. Then $F_{\delta-x x}^{\delta}=f_{-\partial x}$, where:

$$
\begin{aligned}
& f_{1}=1, \\
& f_{2}=X-3, \\
& f_{3}=X^{2}-5 X+7, \\
& f_{4}=X^{3}-6 X^{2}+15 X-14, \\
& f_{5}=X^{4}-7 X^{3}+22 X^{2}-37 X+26, \\
& f_{6}=X^{5}-7 X^{4}+22 X^{3}-45 X^{2}+62 X-39 .
\end{aligned}
$$

\section{The defect of GR submodules in homogeneous modules of dimension $\delta$}

As an application of the results from the previous sections, using Ringel's idea from [13] we can prove the main result from $[\mathbf{6}]$ in a more general setting: the result is valid also for the case $\tilde{E}_{8}$ (this case is missing from $[\mathbf{6}]$ ) and the base field $k$ is arbitrary (in [6] $k$ is algebraically closed).

TheOREM 5.1. Let $Q$ be a tame quiver with minimal radical vector $\delta$. If $R$ is a homogeneous module with dimension $\delta$ and $P$ a GR submodule, then $P$ has defect -1 . As a consequence, the pair $(R / P, P)$ is a Kronecker pair.

Proof. Since in the case $\tilde{A}_{n}$ the defect of a preprojective indecomposables is always -1 , we suppose that $Q$ is not of type $\tilde{A}_{n}$. We have $P=P(x, k)$, where $x$ is a positive real root with $\partial x<0$. Using Theorem 3.10, it follows that $P\left(x, k^{\prime}\right) \rightarrow R^{k^{\prime}}\left(a^{\prime}\right)$ is a GR inclusion for any field $k^{\prime}$ and any point $a^{\prime} \in \mathbb{X}_{k^{\prime}}$. By Corollary 3.11, this means that $F_{\delta-x x}^{\delta}=\left(X^{-\partial x}-X^{s}\right) /(X-1)$. But we also know from Theorem 4.2 that $F_{\delta-x x}^{\delta}=f_{-\partial x}$. Comparing the polynomials, one can see that $\partial x=-1$.

Since $R / P$ is indecomposable preinjective of defect 1 , the second assertion follows using the observations in Section 2.

Using the observations from Section 2, note that if $P=P(x, k)$ is a GR submodule in a homogeneous module $R$ of dimension $\delta$, then $R / P=I(\delta-x, k)$ and the pair $(I(\delta-x, k), P(x, k))$ exists and remains a Kronecker pair even in the case when the field has two elements and the quiver is not of type $\tilde{A}_{n}$ (so we do not have homogeneous modules of dimension $\delta$ ).

Acknowledgements. The authors are very grateful to the referee for suggestions and comments to improve the manuscript.

\section{References}

1. I. Assem, D. Simson and A. Skowronski, Elements of representation theory of associative algebras, volume 1: techniques of representation theory, LMS Student Texts 65 (Cambridge University Press, Cambridge, 2006).

2. M. Auslander, I. Reiten and S. Smalø, Representation theory of Artin algebras, Cambridge Studies in Advanced Mathematics 36 (Cambridge University Press, Cambridge, 1995).

3. I. N. Bernstein, I. M. Gelfand and V. A. Ponomarev, 'Coxeter functors and Gabriel's theorem', Uspiehi Mat. Nauk. 28 (1973) 19-33 (Russian), Russian Math. Surveys 28 (1973) 17-32 (English).

4. P. Caldero and F. Chapoton, 'Cluster algebras as Hall algebras of quiver representations', Comment. Math. Helv. 81 (2006) 595-616.

5. B. Chen, 'Comparison of Auslander-Reiten theory and Gabriel-Roiter measure approach to the categories of tame hereditary algebras', Comm. Algebra 36 (2008) 4186-4200.

6. B. Chen, 'The Gabriel-Roiter submodules of simple homogeneous modules', Proc. Amer. Math. Soc. 138 (2010) 3415-3424. 
7. V. Dlab and C. M. Ringel, 'Indecomposable representations of graphs and algebras', Mem. Amer. Math. Soc. 173 (1976).

8. A. Hubery, 'Hall polynomials for affine quivers', Represent. Theory 14 (2010) 355-378.

9. I. G. MacDonald, Symmetric functions and Hall polynomials (Clarendon Press, Oxford, 1995).

10. C. M. RingeL, 'Hall polynomials for the representation-finite hereditary algebras', Adv. Math. 84 (1990) 137-178.

11. C. M. Ringel, 'Exceptional modules are tree modules', Linear Algebra Appl. 275-276 (1998) 471-493.

12. C. M. Ringel, 'The Gabriel-Roiter measure', Bull. Sci. Math. 129 (2005) 726-748.

13. C. M. Ringel, 'The theorem of Bo Chen and Hall polynomials', Nagoya J. 183 (2006) 143-160.

14. S. V. SAM, 'The Caldero-Chapoton formula for cluster algebras', Preprint, 2009, https://math.berkeley. $\mathrm{edu} / \sim$ svs/talks/calderochapoton.pdf.

15. The GAP Group, 'GAP_-groups, algorithms, and programming, version 4.6.5', 2013, http://www.gapsystem.org.

Csaba Szántó and István Szöllősi

Faculty of Mathematics and Computer Science

Babeş-Bolyai University

Str. Mihail Kogălniceanu, nr. 1

R0-400084 Cluj-Napoca

Romania

szanto.cs@gmail.com

szollosi@gmail.com 